\title{
RESPONSES OF DAMPED HARMONIC OSCILLATORS TO EXCITATIONS OBEYING POISSON DISTRIBUTIONS
}

\author{
HYOUNG-IN LEE* AND JINSIK MOK
}

\begin{abstract}
External excitations are employed to investigate properties of optical media, with measurement data often analyzed via linear response theory. In this respect, external forcing is modeled here by well-known Poisson and negative-binomial distributions. Ensuing dynamics is examined with a special attention to the relative decay rates of damped harmonic oscillators to such external forcing, along with its relationship to other physical phenomena.

AMS Mathematics Subject Classification : 34M10, 60J80, 81V80.

Key words and phrases : Ordinary differential equations, Photon statistics,
\end{abstract} Branching decays, Poisson distributions, Coupled resonators, Plasmonics.

\section{Introduction}

Linear responses of harmonic oscillators to external forcing are very useful to understanding fundamental workings of materials $[1,2]$. In particular, damped harmonic oscillators (DHOs) have been employed to examine various materials ranging from superconductors [1], electronics [3], and plasmonics [4], to name a few. For instance, electric polarizability can be inferred by illuminating lights on optical media. Even with nonlinearity disregarded, linear response theory gets complicated as physics becomes more closely modeled. There are several factors contributing to this increased complexity: (i) the number of oscillators and associated coupling [3, 5], (ii) the presence of damping, and (iii) the types of external forcing. When it comes to DHOs, clear-cut demarcations among these three ingredients get blurred [1]. In particular, optical damping involves a close interplay between a system (if properly definable) and its environment. Energy transfer is sometimes enhanced by damping while energy content itself is diminished [4]. Therefore, damping renders a system non-conservative, for which both classical and quantum mechanical formulations are still imperfect [6].

Received August 23, 2012. Accepted November 20, 2012. ${ }^{*}$ Corresponding author

(c) 2013 Korean SIGCAM and KSCAM. 
In this article, we investigate effects of external forcing on DHOs from a viewpoint of classical mechanics. In particular, we focus on forcing obeying Poisson distributions because excitations modeled even as single square-shaped pulses are distributed with certain decay rates when resolved on finer time scales. Furthermore, multiple exciters can be considered to be composed of a parallel array of time-decaying excitation agents. In this respect, we assume these exciters to obey well-known statistics of either Poisson or negative--binomial distribution [7] - [11]. In particular, the negative-binomial distribution is suitable for describing particle branching processes involving decays [9]. We focus in this article on addressing various physical ramifications of ensuing dynamics under such excitations by either analytical or numerical means.

In the linear response of a DHO to external forcing $f(t)$, a crucial role is played by the relative magnitudes of two time scales. As the first, the decay rate $\gamma$ of a DHO stands, e.g., for inelastic scattering within lossy metals, which occurs in photon-electron interactions often examined in plasmonics [4]. As the second, another decay rate $a$ characterizes forcing, which is here described by Poisson distribution $p_{n}(a t) \equiv(n !)^{-1}(a t)^{n} e^{-a t}$ over a discrete support of integer $n$ with $t$ as time. As regards photon counting in quantum optics, $n$ refers to a finite number of photons. With $\sigma \equiv a t$, its first moment is given by $\bar{n}_{p} \equiv\langle n\rangle_{P}=\sigma$, where the subscript $P$ refers to "Poisson". Beside, the Mandel $Q$-parameter is found to be $Q_{p}=0$ (i.e., Poissonian). For convenience, we divide the decay-rate regime into two: (i) over-decaying excitations with $a>\gamma$, and (ii) underdecaying excitations with $a<\gamma$. Hence, we call the case $a=\gamma$ equally decaying. By a standard double-Poisson distribution, we consider the statistics obtained by varying $a$ over all positive real values, as will be discussed in Section 3 . The over-decaying regime is of particular interest to optical measurements, since responses intrinsic to a system last longer than excitations for $a<\gamma$ and they are amenable to longer experimental observations. In one-atom-maser theory [12], the damping-bases method relies on a similar principle.

\section{Response to forcing obeying Poisson distribution}

Consider mechanical vibrations of a single DHO, where its displacement $x(t)$ is governed by the following differential equation $[1,6]$.

$$
\ddot{x}+2 \gamma \dot{x}+k x=f(t) u\left(t-0_{+}\right)
$$

Here, $\gamma \geq 0$ is damping, $k \equiv \gamma^{2}+b^{2}$ a spring constant, and $b \geq 0$ a quasifrequency. In optics, $x$ refers to electric polarization of a given medium, whereas $f$ means an applied electric field. By a Heaviside unit step function $u\left(t-0_{+}\right)$, $f$ is meant to be applied right after the initial state, so that initial conditions (ICs) $x_{0} \equiv x(0)$ and $\dot{x}_{0} \equiv \dot{x}(0)$ remain valid for any $f$. However, $u\left(t-0_{+}\right)$will be henceforth omitted for simplicity.

Let us define variants of the ICs by $b y_{0} \equiv \dot{z}_{0} \equiv \dot{x}_{0}+\gamma x_{0}$. Then, the solution is written as $x=x_{h}+x_{p}$, with the homogeneous solution (HS) and particular 
solution (PS) denoted respectively by $x_{h}(t)$ and $x_{p}(t)$. It is readily found that $x_{h}(t)=\left(x_{0} \cos (b t)+y_{0} \sin (b t)\right) e^{-\gamma t}$. For $f(t)=p_{n}(a t)$, which might represent radiation pressure exerted by photons [13], $x_{p}(t)$ is found as follows.

$$
x_{p}(t)=\frac{a^{n}}{b n !} \int_{0}^{t}(t-\eta)^{n} e^{-a(t-\eta)} e^{-\gamma \eta} \sin (b \eta) d \eta .
$$

The RHS of Eq.(2) should be multiplied by $n$ to account for a total force. Therefore, $x_{p}$ here can be considered as a displacement in response to, say, a single photon. Figure 1 shows trajectories on the phase plane of $x$ and its velocity $\dot{x}$. To this goal, we have numerically integrated Eq.(2). Panel (a) shows a characteristic spiral trajectory with the clockwise direction of time evolution indicated by arrows, where it approaches a quiescent state at the origin [6].
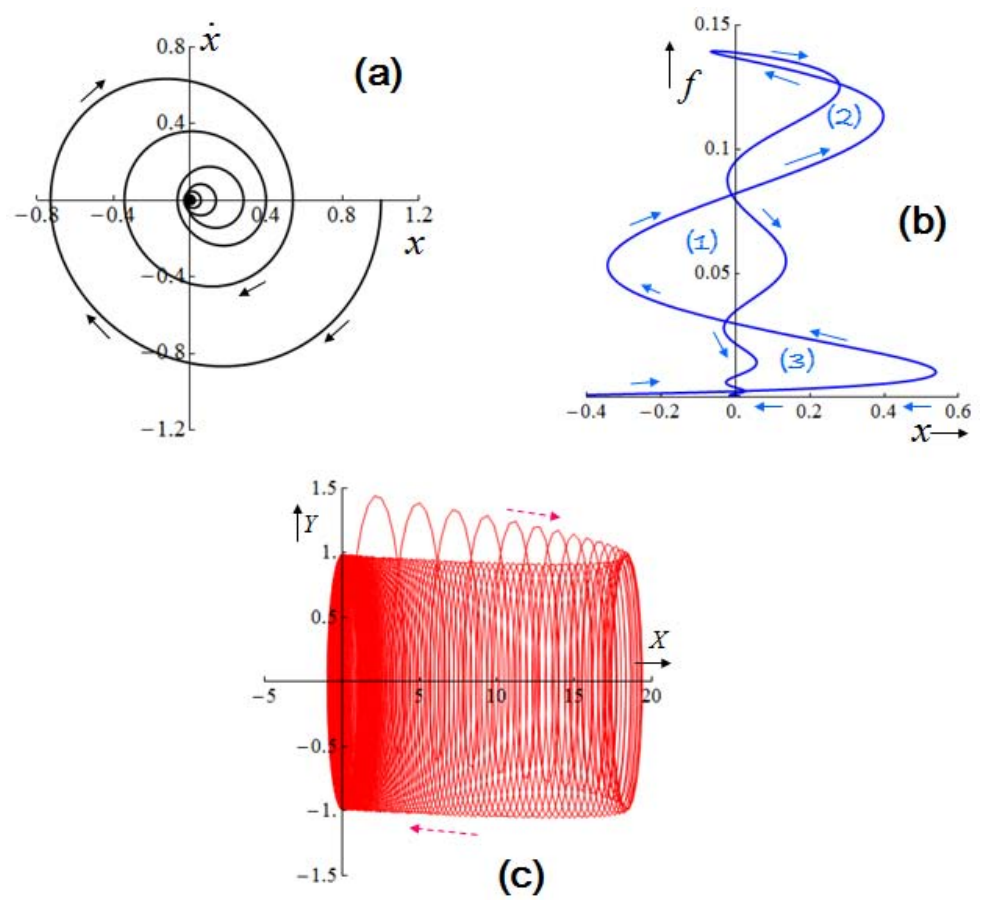

FIGURE 1. For given data $n=8$ and $\gamma=0.1$, solution trajectories on the phase planes: (a) displacement $x$ and velocity $\dot{x}$, and (b) external forcing $f$ vs. normalized displacement $x_{p}$. (c) Solution trajectory on the plane of reduced displacement $X$ and velocity $Y$ for $n=1$ and $\gamma=0.49$. Common prescribed data are $a=0.5, b=1, x_{0}=1$, and $\dot{x}=0$.

Panel (b) is another presentation of the result of Panel (a), where the ordinate is forcing $f$ as input and the abscissa is $x$ as output. The curve starting with $(x, f)=(1,0)$ at $t=0$ (out of the display window) appears to advance in the left 
direction almost along the $x$-axis. Before it approaches $(x, f)=(0,0)$ as $t \rightarrow \infty$, it makes several turns, as indicated by the time arrows. Panel (b) is called a pressure-volume (or force-displacement) diagram often employed in quantum opto-mechanics [13]. On the other hand, Panel (c) shows $Y(t) \equiv y(t) e^{\gamma t}$ versus $X(t) \equiv x(t) e^{\gamma t}$, where $b y \equiv \dot{x}+\gamma x$, such that the decay-compensating factor $e^{\gamma t}$ is multiplied. The broken line in Panel (c) shows a "bounce"-type envelope of the trajectory with a turning point approximately at $X=18$ [1].

From energy consideration of Eq.1, $\dot{U}+\dot{K}=\dot{x} f-4 \gamma K$ with the potential energy $U \equiv 0.5 x^{2}$ and kinetic energy $K \equiv 0.5 \dot{x}^{2}$. Hence, the total work done is defined by $W \equiv W_{h}+W_{p}$, where $\left\{W_{h}, W_{p}\right\} \equiv \int_{0}^{\infty} f(t)\left\{\dot{x}_{h}(t), \dot{x}_{p}(t)\right\} d t$ in correspondence to the homogeneous and particular displacements. Numerically, $W$ is equal to the net area enclosed by the clockwise trajectories of a curve on Figure 1(b), namely, the area(1)-area(2)-area(3) in rough estimate. Besides, the total impulse $\int_{0}^{\infty} f(t) d t$ is $a^{-1} n$ for $f(t)=p_{n}(a t)$. With $\rho^{2} \equiv(\gamma+a)^{2}+b^{2}$ and $\tan \left(\theta_{0}\right) \equiv b /(a+\gamma)$, we employ contour integrals to find

$$
W_{h}=\frac{a^{n}}{\rho^{n+1}}\left\{\dot{x}_{0} \cos \left((n+1) \theta_{0}\right)-b^{-1}\left\lfloor\left(\gamma^{2}+b^{2}\right) x_{0}+\gamma \dot{x_{0}}\right\rfloor \sin \left((n+1) \theta_{0}\right)\right\} .
$$

For $W_{h}=0$, there is a constraint among $\dot{x_{0}} / b x_{0}$ of the ICs, $\gamma / b$, and $a / b$.

Through another double integration by numerical means, we obtain

$$
W_{p}=\frac{a^{2 n}}{b(n !)^{2}} \int_{0}^{\infty} t^{n} e^{-2 a t}\left\lfloor\int_{0}^{t}(t-\tau)^{n} e^{(a-\gamma) \tau} \sin (b \tau) d \tau\right\rfloor d t
$$

Figure 2 (a) plotting $W_{p}$ against $n$ shows that $W<0$ for low $n$-values, whereas $W>0$ for high $n$-values. Here, we find almost monotonic decreases in $|W|$ with increasing $n$, irrespectively of $a$. Following the idea employed in meteoric observations [14], Figure 2(b) shows $\varpi(n) \equiv|W(n+1) / W(n)|$ between two successive works done, which turns out fairly constant for larger $n$-values. However, the behaviors at small $n$-values are strongly dependent on the ICs among various parameters.

Let us consider a few special cases. In an equally decaying case that $\gamma=a$ together with $b \neq 0$, we have $x_{h}(t) \propto e^{-\gamma t}$ and $x_{p}(t)=(b n !)^{-1} \gamma^{n} e^{-\gamma t} \int_{0}^{t}(t-$ $\eta)^{n} \sin (b \eta) d \eta$ from Eq.(2). In a still further restrictive case that $\gamma=a$ and $b=0, p_{n}(\gamma t)=(n !)^{-1}(\gamma t)^{n} e^{-\gamma t}$ and we obtain only a single-term response $x_{p}(t)=((n+2) !)^{-1} \gamma^{n} t^{n+2} e^{-\gamma t}$ with a 2 -step overtone. We could exploit this very special excitation to measure the damping $\gamma=a$ by properly analyzing output signals. For work done, $W_{p}=\gamma^{-2} 2^{-2 n-3}(n !(n+2) !)^{-1}(2 n+2)$ in this case.

On the other hand, in the evanescent-wave-like case that $b^{2} \equiv-\bar{b}^{2}<0$ and $k \equiv \gamma^{2}-\bar{b}^{2}$ with $0 \leq \bar{b} \leq \gamma$, we obtain $x_{h}=\left\lfloor x_{0} \cosh (\bar{b} t)+\bar{b}^{-1} z_{0} \sinh (\bar{b} t)\right\rfloor e^{-\gamma t}$. In addition, Eq. (2) is changed for $n>0$ into $x_{p}(t) \equiv(\bar{b} n !)^{-1} a^{n} e^{-a t} \int_{0}^{t}(t-$ $\eta)^{n} e^{-(a-\gamma) \eta} \sinh (\bar{b} \eta) d \eta$ in this case. Let us now examine another limit $\bar{b}=\gamma$, for which $k=0$ in Eq.(1), thus meaning no restoring force as is the case with free electrons under consideration in plasmonics [4]. In terms of electric-circuit, 

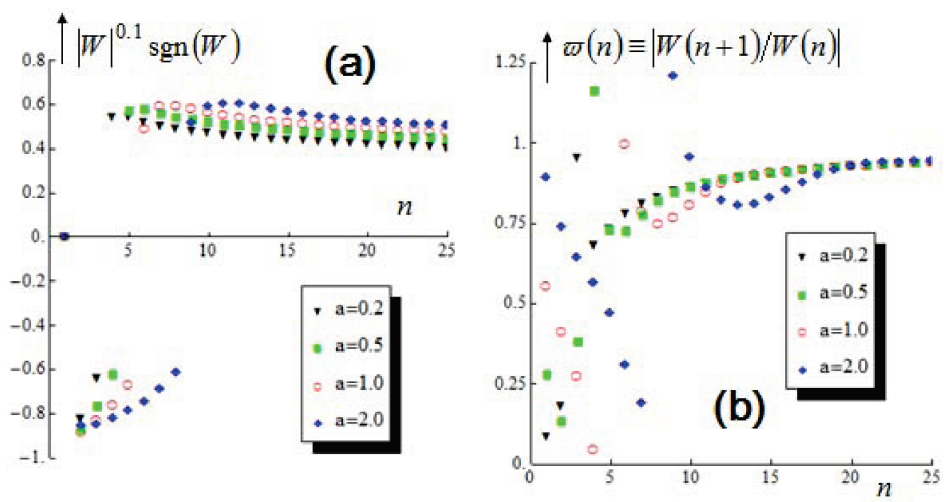

FiguRE 2. (a) Signed work done $W$ vs. $n$ for four $a$-values, with a scaling $|W|^{0.1} \operatorname{sgn}(W)$ for better viewing contrast. (b) Ratio of works done $\varpi(n)$. Common prescribed data are $\gamma=1, b=1$, and $a=0.5$, along with $x_{0}=1$ and $\dot{x}_{0}=0$.

$k=0$ corresponds to an infinite capacitance. Because the LHS of Eq.(1) reduces now to $\ddot{x}+2 \gamma \dot{x}$, the equal decay takes place not when $a=\gamma$, but when $a=$ $2 \gamma$. In this case, Eq. (2) is integrated to $x_{p}(t)=(2 \gamma)^{-2}\left\lfloor(n !)^{-1} \int_{0}^{2 \gamma t} \eta^{n} e^{-\eta} d \eta-\right.$ $\left.((n+1) !)^{-1}(2 \gamma t)^{n+1} e^{-2 \gamma t}\right\rfloor$. Consequently, we have $\lim _{t \rightarrow \infty} x_{p}=0.25 \gamma^{-2}>0$. Besides, $x_{h}(t)=0.5 x_{0}\left(1+e^{-2 \gamma t}\right)+0.5 \gamma^{-1} z_{0}\left(1-e^{-2 \gamma t}\right)$.

As yet another special case, consider $f(t)=\sum_{n=0}^{\infty} p_{n}(a t)=1$ accounting for all the possible number of occurrences. It is like the situation where small particles or large meteors of all sizes are taken into account in the respective fields of physics dealing with aggregates or clusters [8]. In our present case, $x=x_{\infty}+\left(x_{0}-1\right) \cos (b t) e^{-\gamma t}+b^{-1}\left(\gamma\left(x_{0}-1\right)+\dot{x}_{0}\right) \sin (b t) e^{-\gamma t}$, with $x_{\infty} \equiv\left(\gamma^{2}+b^{2}\right)^{-1}$. Hence, starting out with $x_{0}, \lim _{t \rightarrow \infty} x(t)=x_{\infty}$. With this kind of summation, both single Poisson and negative-binomial distributions (for the latter, see next section) can be associated with an infinite number of damped harmonic oscillators. This concept is important, since external forcing can constitute a whole system along with the other two partners, namely, a DHO-subsystem and its environment [1]. The coupling and energy transfer among such DHOs will make the subject of our forthcoming article.

\section{Responses to forcing of negative-binomial distribution}

From physical viewpoint, the decay rate itself is not necessarily fixed. We could model distributed decay rates with the help of another single-Poisson distribution [7]. We are hence to examine a negative-binomial (NB) distribution, which exhibits an algebraic or sub-exponential decay in comparison to 
the exponential decay characterized by a single-Poisson distribution [8]. Interestingly enough, delayed luminescence discussed in biophotonics [10] is adequately explained by such a NB distribution, because of hyperbolic-like longterm afterglow in living systems. Consider a gamma distribution $g_{\mu}(a, T) \equiv$ $(\Gamma(\mu))^{-1} T^{\mu} a^{\mu-1} e^{-T a}$, where both $\mu$ and $T$ are real-valued. In addition, the corresponding average decay rate or its first moment is $\bar{a} \equiv\langle a\rangle_{G}=T^{-1} \mu$, where the subscript $G$ refers to "Gamma". With $g_{\mu}(a, T)$ and another scaled time $\tau \equiv T^{-1} t$, we define a NB distribution $g_{\mu}(a, T)$ as follows.

$$
q_{\mu, n}(\tau) \equiv \int_{0}^{\infty} g_{\mu}(a, T) p_{n}(a t) d a=\frac{\Gamma(n+\mu)}{n ! \Gamma(\mu)} \frac{\tau^{n}}{(1+\tau)^{n+\mu}} .
$$

The average of $n$ taken with respect to $q_{\mu, t}(\tau)$ is $\bar{n}_{N B} \equiv\langle n\rangle_{N B}=\mu \tau$ [8]. The corresponding Mandel $Q$-factor is analytically found to be $Q_{N B}=\tau$. Consequently, $q_{\mu, n}(\tau)$ is super-Poissonian as is true with classical photon counting. With a particular prescribed data $\mu=\gamma, q_{\mu, n}(\tau)$ looks very similar to $p_{n}(\sigma)$ (not shown). Figure 3(a) shows the resulting $x(t)$ in response to $f(t)=q_{\mu, n}\left(T^{-1} t\right)$ with rather high damping. In comparison, Figure 3(b) shows responses to the forcing with relatively small damping. We found from dynamics among $q_{\mu, n}(\tau)$ 's with continuous $\mu$ that there are waves traveling on the $\tau-\mu$ plane, which will be discussed in a separate article.
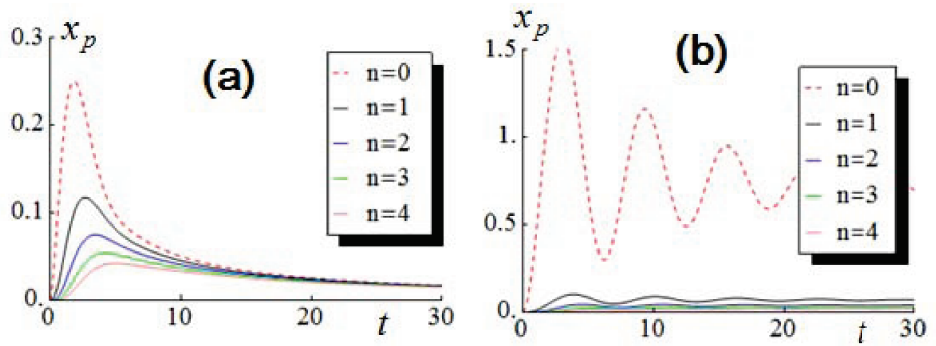

FiguRE 3. Particular solutions for (a) high damping with $\mu=\gamma=1$ and (b) low damping with $\mu=\gamma=0.1$. Common data are $b=1$ and $T=1$, along with $x_{0}=1$, and $\dot{x}_{0}=0$.

\section{Discussions and conclusion}

To assess the importance of the net gain $\Delta \equiv a-\gamma$, we could formulate problem with Eq.(1) in two different manners along the reasoning of the damping-bases method in quantum optics [12]. Let us consider a displacement $z(t) \equiv x(t) e^{a t}$ from the external-decay-based (EDB) viewpoint. Its homogeneous solution is then reduced to $z_{h}(t)=\left(x_{0} \cos (b t)+y_{0} \sin (b t)\right) e^{\Delta t}$. In addition, its particular solution $z_{p}$ is determined from Eq.(1) by solving $\ddot{z}_{p}-2 \Delta \dot{z}_{p}+\kappa z_{p}=$ $f(t)=(n !)^{-1}(a t)^{n}$. Therefore, from this EDB viewpoint, the parameter regimes $\gamma>a$ and $\gamma<a$ refer to the loss and gain, respectively. In comparison to the 
original spring constant $k, \kappa \equiv \Delta^{2}+b^{2}$ is an effective spring constant from this EDB viewpoint. It is found then that $\kappa<k$ as long as $0<a<2 \gamma$, thereby being terms "softening" of a spring. In the remaining regime $2 \gamma<a, \kappa>k$, thus signifying "hardening".

Equation (1) is equivalent to a pair of coupled equations $\dot{y}=-k x$ and $\dot{x}=y-$ $2 \gamma x+\int_{0+}^{t} f(\tau) d \tau$, where $x$ and $y$ refer to the system and environmental variables, respectively. It is clear from an auxiliary equation $\ddot{y}+k y=k\left\lfloor 2 \gamma x-\int_{0_{+}}^{t} f(\tau) d \tau\right\rfloor$ that energy is irreversibly lost from $x$ to $y[1]$. In this regard, the condition for $x$ to be of Poisson distribution will be interesting, given that forcing upon $x$ is of Poisson distribution. Incidentally, we might need a vector equation in comparison to the scalar one in Eq.(1), in order to take light polarization into account.

In summary, we have examined Poisson statistics for applied excitations exerted on damped harmonic oscillators. By this way, we have been led to find a central role played by two competing decay rates (intrinsic vs. external), which in turn motivated us to consider distributed decay rates by negative-binomial statistics. In addition, several ramifications of the current results have been explained in terms of experimental measurements.

\section{Acknowledgments}

This research has been supported by Basic Science Research Program through the National Research Foundation (NRF) of Korea funded by the Ministry of Education, Science and Technology (Grant Number: 2011-0023612). We have greatly benefited from discussions with Professors. Jin-O Kim (Soongsil Univ.) on excitations, Jaewan Kim (KIAS) on probability, and Seung-Yeal Ha (SNU) on synchronization.

\section{REFERENCES}

1. A.O. Caldeira, and A. J. Leggett, "Quantum tunneling in a dissipative syste", Ann. Phys. 149 (1983) 374-456

2. U. Weiss, Quantum Dissipative Systems, Third Edition, World Scientific, Singapore (2008)

3. L. Novotny, "Strong coupling, energy splitting, and level crossings: A classical perspective", Am. J. of Physics 78 (2010) 1199

4. H.-I. Lee, and E.-H. Lee, "Complex relaxation rates of the Drude metals and their effects on the lifetime and symmetry of plasmon resonances", Opt. Ex. 19 (2011) 10410-10422

5. Y.-P. Choi, S.-Y. Ha, S.-B. Yun, "Complete synchronization of Kuramoto oscillators with finite inertia", Physica D 240 (2011) 32-44

6. N. W. Hone, and M. Senthilvelan, "Note on the Poisson structure of the damped oscillator", J. Math. Phys. 50 (2009) 102902

7. M. C. Teich, and B. E. A. Saleh, "Observation of sub-Poisson Franck-Hertz light at 253.7 nm”, J. Opt. Soc. Am. B 2 (1985) 275-282

8. P. Carruthers, and C. C. Shih, "The phenomenological analysis of hadronic multiplicity distributions", Int. J. of Modern Physics A 2 (1987) 1447-1547

9. N. Suzuki, M. Biyajima, and G. Wilk, "Stochastic background of negative binomial distribution", Physics Letters B 268 (1991) 447-452 
10. F. A Popp, J. J. Chang, A. Herzog, Z. Yan, and Y. Yan, "Evidence of non-classical (squeezed) light in biological systems", Physics Letters A 293 (2002) 98-102

11. B. Markussen, Inference for Stochastic Partial Differential Equations and Chaos Decomposition of the Negative Binomial Process, Ph.D. thesis, University of Copenhagen (2002)

12. Hans-Jrgen Briegel, and Berthold-Georg Englert, "Quantum optical master equations: The use of damping bases", Phys. Rev. A 47 (1993) 3311-3329

13. T. J. Kippenberg, and K. J. Vahala, "Cavity Opto-Mechanics", Opt. Ex. 15 (2007) 1717217205

14. S. van den Bergh, "Magnitudes of Sporadic and Perseid Meteors", Meteoritics 1 (1956) 395-398

Hyoung-In Lee received both B.S. (1979) and M.S. (1981) from the Department of Mechanical Engineering, Seoul National University, and his Ph.D. from the Dept. of Theoretical and Applied Mechanics (TAM), the University of Illinois at Urbana-Champaign (UIUC) in USA (1988). Thereupon, he spent two years as a postdoctoral fellow at UIUC. In 1990, he went on to the Samsung Advanced Institute of Technology (SAIT), which belongs to Samsung Electronics in Korea. During 17 years at SAIT, he has been involved in various computational-science projects for electronics products such as optical fibers, bulk crystals, optical waveguides, plasma processing for memory devices, to name a few. From 2007 to 2011, he has been with Optics and Photonics Elite Research Academy (OPERA) of Inha University in Korea. Currently, he concerns himself with superconducting photonic crystals and plasmonics.

Research Institute of Mathematics, Seoul National University, Seoul, 151-747 Korea.

e-mail: hileesam@naver.com

Jinsik Mok received her B.S. from the Dept. of Mathematics Education, Seoul National Universityand her M.S. (1987) and Ph.D. (1992) from Mathematics Dept. at UIUC in USA. Her major field of research lies in analysis and probability.

Department of Mathematics, Sunmoon University, Asan, Chungnam 336-708 Korea

e-mail: jsmok@sunmoon.ac.kr 